\title{
Afebrile patients with severe acute respiratory syndrome coronavirus 2 infection have a longer viral positivity duration: a retrospective analysis of 143 patients
}

\section{Rui Ding}

Capital Medical University Affiliated Beijing Ditan Hospital

\section{Rugang Zhao}

Capital Medical University Affiliated Beijing Ditan Hospital

Wen Xie ( $\nabla$ xiewen6218@163.com )

Ying Fan

Capital Medical University Affiliated Beijing Ditan Hospital

Ligai Liu

Capital Medical University Affiliated Beijing Ditan Hospital

\section{Ying Cao}

Capital Medical University Affiliated Beijing Ditan Hospital

\section{Cheng Cheng}

Capital Medical University Affiliated Beijing Ditan Hospital

\section{JingJing Wang}

Capital Medical University Affiliated Beijing Ditan Hospital

\section{Qi Wang}

Capital Medical University Affiliated Beijing Ditan Hospital

\section{Yanbin Wang}

Capital Medical University Affiliated Beijing Ditan Hospital

\section{Ting Zhang}

Capital Medical University Affiliated Beijing Ditan Hospital

Minghui LI

Capital Medical University Affiliated Beijing Ditan Hospital

\section{Research}

Keywords: Afebrile, febrile, SARS-CoV-2, duration of positivity, risk factors

Posted Date: September 11th, 2020 
DOl: https://doi.org/10.21203/rs.3.rs-36961/v2

License: (c) (1) This work is licensed under a Creative Commons Attribution 4.0 International License. Read Full License 


\section{Abstract}

Background A pandemic of severe acute respiratory syndrome coronavirus 2 (SARS-CoV-2) is on-going. Clinical characters of afebrile cases infected with SARS-CoV-2 remain poorly understood and informations are limited on the duration of SARS-CoV-2 viral positivity.

Methods We performed a single-center retrospective study of 143 patients with SARS-CoV-2 infection in Beijing Ditan Hospital, Capital Medical University from January 26 to April 15, 2020. Differences were compared among patients with/without fever. Risk factors for the duration of SARS-CoV-2 viral positivity were evaluated.

Results A total of 143 patients with positive SARS-CoV-2 test were enrolled, including 38 afebrile patients and 105 febrile patients. On admission, a total of $40(28 \%)$ patients had leukopenia, $44(30.8 \%)$ had lymphopenia and $8(5.6 \%)$ had thrombocytopenia. 78 patients $(54.5 \%)$ had decreased T lymphocytes and 105 patients $(73.4 \%)$ had decreased CD4+T lymphocytes. Compared with febrile cases, afebrile patients had a significantly higher white blood cell count $(P=0.02)$, total lymphocytes $(P<0.01)$, platelet count $(P$ $<0.01)$, T lymphocytes $(P<0.01)$ and CD8+ T lymphocytes $(P=0.02)$. The median SARS-CoV-2 viral positivity duration of these 143 patients was 14 days (IQR, 10-30 days) and for febrile and afebrile group were 13 days (IQR, 10-29 days) and 20 days (IQR, 11-31 days) respectively. Multivariate Cox regression results showed that the fever [hazard ratio $(\mathrm{HR})=0.49, P<0.01$ ] and higher count of platelet $(\mathrm{HR}=5.47, P$ $=0.02$ ) were the predominant risk factor for the SARS-CoV-2 viral positivity duration.

Conclusion The SARS-CoV-2 virial positivity duration of the afebrile group was significantly longer than that in the febrile group. Fever and a higher count of platelet were the independent protective factors for a shorter SARS-CoV-2 RNA positivity duration.

\section{Background}

The infection of severe acute respiratory syndrome coronavirus 2 (SARS-CoV-2) has become a pandemic rapidly. On February 11,2020 , World Health Organization (WHO) officially named the disease of the novel coronavirus disease 2019 (COVID-19) [1]. By the end of the March, more than 7 million people worldwide have been diagnosed with the SARS-CoV-2 infection [2], which brought huge challenges and threats for both global health and economic development. Fever as the main clinical manifestation of SARS-CoV-2 infection is widely used in community and hospital emergency screening $[3,4,5]$. However, we found that afebrile patients of SARS-CoV-2 infection count for a certain part in the process of diagnosis and treatment. What's more, the clinical characteristics and outcomes of these afebrile patients were rarely described until now. In this study, we did a retrospective study to compare the clinical characteristics and SARS-CoV-2 ribonucleic acid (RNA) positivity duration between afebrile and febrile patients with SARS-CoV-2 infection in Beijing Ditan Hospital. Besides, risk factors including clinical and laboratory data for the duration of SARS-CoV-2 RNA positivity were evaluated in 143 patients with SARSCoV-2 infection. 


\section{Methods}

\section{Participants}

From January 26, 2020 to April 15, 2020, a total of 237 patients were hospitalized and confirmed with SARS-CoV-2 infection in Beijing Ditan Hospital, Capital Medical University. We compared the characteristics between febrile and afebrile confirmed cases. For inclusion in the analysis, patients must be $\geq 18$ years of age. Exclusion criteria: (1) Patients who were still hospitalized in Beijing Ditan Hospital, Capital Medical University until May 15, 2020. (2) The patients did not have complete clinical data, and in particular complete blood tests and peripheral blood T-lymphocyte subsets were not performed within 24 hours after admission. (3) Patients who had taken nonsteroidal anti-inflammatory drugs (NSAIDs) including aspirin, ibuprofen, acetaminophen and salicylate from herbal preps before admission.

\section{Definition}

The diagnosis and classification of COVID-19 was based on the latest Diagnosis and Treatment Guidelines for COVID-19 (Trial seventh version) [6]. A confirmed case of COVID-19 was defined as a positive result on high throughput seguencing or real-time reverse-transcriptase-polymerase-chainreaction (RT-PCR) assay of nasal and pharyngeal swab specimens [7]. Mild type was defined as having minimal symptoms and negative CT findings. Common type was defined as having fever, respiratory tract and other symptoms with pneumonia in imaging. Severe type was defined as the presence of respiratory distress, respiratory rate $\geq 30$ times/min; oxygen saturation (resting state) $\leq 93 \%$; $\mathrm{PaO} 2 / \mathrm{FiO} 2 \leq$ $300 \mathrm{mmHg}$. Critical type was defined as the presence of respiratory failure requiring mechanical ventilation, shock and other organ failure requiring ICU monitoring and treatment. In this study, we assessed the degree of severity of COVID-19 as mild (mild and ordinary type) vs. severe (severe and critical type) cases at the time of admission according to their condition mentioned above. Afebrile state was defined as an axillary temperature less than $37.3^{\circ} \mathrm{C}$ in the course of COVID-19. Leukopenia was defined as a white blood count of less than 4000 cells per cubic millimeter. Lymphocytopenia was defined as a lymphocyte count of less than 1000 cells per cubic millimeter. Thrombocytopenia was defined as a platelet count of less than 100,000 per cubic millimeter.

\section{Data collection}

Specimens of the nasopharyngeal swab samples were collected every other day during hospitalization. SARS-CoV-2 RNA positivity duration time was calculated by an interval from the first day with SARS-CoV2 RNA tested positive to the first day of SARS-CoV-2 returned to negative (at least consecutive two times of the RT-PCR negative results). The following parameters were collected from electronic medical records: (1) General conditions: gender, age, symptoms, time from illness onset to hospital admission and severity of illness; (2) Chest CT findings within 24 hours after admission; (3) Laboratory characteristics: white 
blood cell, neutrophil, lymphocyte, monocyte, red blood cell, hemoglobin, platelet, fibrinogen, D-dimers, $T$ lymphocyte, CD4 + T lymphocyte, CD8 + T lymphocyte, NK cell, B lymphocyte, albumin, lactic dehydrogenase, C-reactive protein, erythrocyte sedimentation rate, lactic acid, creatinine, sodium, potassium; (4) Clinical outcomes: duration of SARS-CoV-2 RNA positivity and length of hospital stay. The purpose of this study is to explore the characteristics of afebrile patients and observe the risk factors of viral RNA clearance within 30 days after illness onset.

\section{Statistical analysis}

The statistical analysis was performed utilizing SPSS software (Version 19.0). Continuous variables were expressed as median with interquartile range (IQR) and compared by Mann-Whitney U test. Categorical variables were expressed as number (\%) and compared by Chisquare $\left(\chi^{2}\right)$ test or Fisher's exact test between groups. Cox regression proportional-hazards model was applied to assess the hazard ratio (HR) of each variable for the duration of SARS-CoV-2 viral positivity, and significant risk factors whose HR was further adjusted with covariate analysis. All statistical tests were two-sided and a $P<0.05$ was considered statistically significant. Kaplan-Meier method with a Log-rank test was performed to evaluate the significance of the duration of SARS-CoV-2 viral positivity between afebrile and febrile group.

\section{Results}

\section{The selection process of included patients}

A total of 143 patients with confirmed SARS-CoV-2 infection were included in this study from January 26 to April 15, 2020 at Beijing Ditan Hospital, Capital Medical University, including 38 afebrile patients and 105 febrile patients. An overview of the selection process is presented in Figure 1.

\section{Clinical characteristics of afebrile and febrile patients with SARS-CoV-2 infection at admission}

There were no differences in age and gender between the two groups. Myalgia $(P=0.01)$ was more common in the febrile group than the afebrile group. There were no significant differences in respiratory symptoms including cough, sputum, shortness of breath, sore throat and rhinorrhea. 11 patients $(11 / 38$, $29.0 \%)$ in the afebrile group showed normal chest CT on admission while 12 patients $(12 / 105,11.4 \%)$ in the febrile group showed normal chest CT on admission, the difference was statistically significant $(P=$ 0.01). For overall patients, the median time from symptom appearance to hospital admission was 10 days (IQR, 6-13 days) and which had no significant difference between the two groups $(P=0.63)$.

Besides, no significant difference was observed in the proportion of severe cases at admission $(P=0.24)$ (Table 1).

The laboratory examination of the 143 patients is shown in Table 2. On admission, a total of $40(28.0 \%)$ patients had leukopenia, $44(30.8 \%)$ had lymphopenia and $8(5.6 \%)$ had thrombocytopenia. 78 patients (54.5\%) with SARS-CoV-2 infection in this study had a dramatic loss of T lymphocytes, while 60 patients (42.0\%) had decreased CD8+T lymphocytes and up to 105 patients (73.4\%) had decreased CD4+T 
lymphocytes. Compared with febrile cases, afebrile patients had a significantly higher white blood cells $(P$ $=0.02)$, total lymphocytes $(P<0.01)$, platelet count $(P<0.01)$, T lymphocytes $(P<0.01), \mathrm{CD} 4+\mathrm{T}$ cells $(P$ $<0.01)$ and CD8+ T cells $(P=0.02)$. What's more, the plasma levels of $\mathrm{C}$-reactive protein concentrations $(P<0.01)$ and sedimentation rate $(P=0.04)$ were lower significantly in afebrile patients than those in febrile patients. No significant differences were observed in NK cells $(P=0.93)$ and B lymphocytes $(P=$ $0.67)$ between two groups. The median hospitalization time of 143 patients in our study was 21 days (IQR, 16-30 days). There was no statistical difference in length of hospital stay between two groups $(P=$ $0.79)$.

\section{Risk factors related to the duration of SARS-CoV-2 viral positivity}

The median SARS-CoV-2 viral positivity duration of these 143 patients was 14 days (IQR, 10-30 days) and for febrile and afebrile group were 12 days (IQR, 10-26 days) and 20 days (IQR, 11-30 days) respectively. The SARS-CoV-2 RNA positivity duration of the afebrile group was significantly longer than that in the febrile group $(P=0.03)$ (Table 2). For the treatment of the 143 patients, 73 cases $(51.1 \%)$ received interferon- $\alpha$ inhalation, 24 cases $(16.8 \%)$ received oral lopinavir/ritonavir. 35 patients $(24.5 \%)$ received lopinavir/ritonavir combined with interferon- $\alpha$ and 11 patients $(7.7 \%)$ were treated with chloroquine combined with interferon- $a$. There were no statistical differences in treatments between two groups (Table 3).

To observe the occurrence of viral RNA clearance within 30 days after illness onset, univariate Cox regression proportional hazards model was used to evaluate the clinical and laboratory parameters on admission and the results showed a significantly shorter SARS-CoV-2 viral positivity duration was related to younger $(\mathrm{HR}=0.99, P=0.03)$, fever $(\mathrm{HR}=0.54, P=0.01)$ and higher count of platelet $(\mathrm{HR}=4.39, P=$ 0.04). Multivariate Cox regression model showed fever $(H R=0.49, P<0.01)$ and higher count of platelet (HR $=5.47, P=0.02)$ were the predominant protective factors for a shorter SARS-CoV-2 RNA positivity duration (Table 4). Kaplan-Meier with Log-rank method was performed to evaluate the significance of fever to the SARS-CoV-2 viral positivity duration (Figure 2).

\section{Discussion}

The WHO had defined COVID-19 as a pandemic on 11 March 202 [8], as a kind of coronavirus, the infectious number of SARS-CoV-2 were much higher than SARS-CoV in 2003 and MERS-CoV in 2012 $[9,10]$. Temperature monitoring is currently widely used in community and hospital emergency settings as a major means of screening for SARS-CoV-2 infection, but a recent study has shown that the absence of fever in SARS-CoV-2 infection (42.8\%) is much more frequent than that in SARS-CoV (1\%) and MERS-CoV infection $(2 \%)[9,10]$. Until now, reports on the clinical features of these non-febrile patients are rare. In this study, afebrile patients with SARS-CoV-2 infection accounted for $26.6 \%(38 / 143)$, which was lower than 
the previous studies $[3,11]$. The different incidence rates may be explained by the different study populations, different definitions of fever, different data and different prevalence among cities.

Fever is an evolutionarily conserved response of infection or injury and confers survival benefits during damage. When the pathogen enters the body, the first cells to detect foreign antigens are lymphocytes, which produce cytokines, such as interleukins- 1 beta and tumor necrosis factor-alpha, to stimulate other immune cells and begin phagocytosis of invading organisms [12,13]. In the early stages of phagocytosis, endogenous pyrogens are released, causing fever by raising the hypothalamic temperature-setting point in the anterior hypothalamus. Fever, as a consequence of infection and inflammation, also reduces the activity of pathogens, providing a more appropriate working environment for macrophages, lymphocytes and other immune cells, and it is now thought to be a protective response that can accelerate tissue recovery [14]. Emerging evidence suggests that the increase in body temperature during fever is associated with the resolution of many infections and confers a survival advantage [15]. In addition, fever enhances immune surveillance by promoting lymphocyte trafficking to lymphoid organs and inflamed tissues during inflammation $[15,16]$. That may explain the peripheral blood lymphocyte count in the febrile group was significantly lower than that in the afebrile group in our study. However, little is known about the regulation of lymphocyte by fever. In our study, the positive duration of SARS-CoV-2 RNA in febrile patients was significantly shorter than that in afebrile patients, and fever was markedly related to a shorter SARS-CoV-2 RNA positivity duration. Accordingly, we first propose that fever may play a protective role during the process of SARS-CoV-2 infection.

Our study also found that patients in the febrile group had significantly lower platelet counts than those in the non-febrile group. However, patients with a higher count of platelet have a shorter duration of SARS-CoV-2 virial positivity. Beyond the main function in hemostasis and blood coagulation, platelets are currently thought to be an important component of the inflammatory and immune response in the processes of viral infections [17]. It is susceptible to activation, damage, or degradation during severe infections or immune responses [18]. Thrombocytopenia was documented in $44.8 \%$ of the SARS patients on presentation which was higher than our results in patients infected with SARS-CoV-2 [19]. Suppression of bone marrow stromal cells, immune destruction, diffuse intravascular coagulation, platelet chemotaxis and peripheral migration, or phagocytosis by macrophages may be the potential mechanisms of the thrombocytopenia during viral infection [20]. Increasing evidence supports the idea that platelets play a role in host defense against infections. Like traditional innate immune cells, platelets are mobilized adaptively from the bone marrow in response to infection and inflammation, being the earliest and most abundant cells preset at vascular sites of inflammation and release a broad-ranging of immune mediators microparticles and exosomes that modulate innate and adaptive immune cells [21]. Evidences suggest that platelets could interact with viral pathogens directly [22,23]. These functions are achieved through direct interaction with leukocytes, endothelial cells and via the release of soluble inflammatory mediators that enhance recruitment and activation of leukocytes $[24,25]$. Besides, platelets also involve in phagocytosis by enhancing antigen presentation by antigen-presenting cells $[26,27]$. The role of platelets in the clearance of virus was observed in respiratory syncytial virus infection by internalizing viral particles and by enhancing type I IFN production from peripheral blood mononuclear cells [28]. Studies 
had shown that patients with thrombocytopenia in infectious diseases had a higher disease activity [29]. We thus propose that low platelets induced immunodeficiency in SARS-CoV-2 infection in part explain the negative predictive value of low or declining platelet count in our study. Increasing the understanding of immunoregulatory functions of platelets in viral infections will undoubtedly improve our knowledge on disease pathogenesis, clinical management, and therapeutic options.

In our study, leukopenia and lymphocytopenia occurred in 28.0\% (40/143) and 30.8\% (44/143) respectively. Lymphocytopenia was often detected in the infection of SARS-CoV [30], the exact mechanism is still being unclear. A study form Raymond SMW showed a significant decrease in white blood count and lymphocyte during the acute phase of SARS-CoV infection was found in $64 \%$ and $98 \%$ of patients respectively [31]. Compared with SARS-CoV, patients infected with MERS-CoV have a relatively low probability of leukopenia (14\%) and lymphopenia (34\%) [32]. Previous studies also showed that viral infections can lead to a down-regulation of lymphocyte subsets [33]. More than $80 \%$ of patients have a decrease in CD4 + T and CD8 + T lymphocyte counts during the acute phase of SARS-CoV infection $[34,35]$. In a recent study by Fan Wang et al., significant decreases in lymphocytes and their subsets were also observed in patients with SARS-Cov-2 infection [36], suggesting that SARS-CoV-2 infection may have a similar immunologic response to SARS infection. Although coronavirus is not known to productively infect $\mathrm{T}$ lymphocytes, altered antigen-presenting cell function and impaired dendritic cell migration resulting in reduced priming of $T$ lymphocytes likely contribute to a fewer number of $T$ lymphocytes [37]. Despite extensive efforts, there is limited information available on the role of the antigen-specific $T$ cellmediated immune response to coronavirus including SARS-CoV-2.

Due to the sudden outbreak, the time of exposure to the patient's antigen and the onset of symptoms is relatively clear, we believe that this virus is the first infection in these patients, and the immune response is also the primary immune response. This study provided data on the relationship between fever and viral clearance time of SARS-CoV-2 infection, and the result shows that the SARS-CoV-2 RNA positivity duration was significantly longer than that of the febrile patients. A higher count of platelet and fever are independent risk factors for the duration of SARS-CoV-2 nucleic acid positivity. Several limitations to this study must be acknowledged. First, it is a retrospective analysis of the data collected from a single center and, for this reason, our results may be biased. Second, the positive rate may be different between nasal swabs samples and sputum [38], so the bias might be introduced when comparing differences directly in viral RNA shedding between sputum versus nasal swabs. A large-scale cohort study and multi-center clinical data are still needed to further elucidate the features of SARS-CoV-2 infection and achieve a better understanding of the interactions between the virus and host response.

\section{Conclusions}

About a quarter of patients with SARS-CoV-2 infection did not experience fever in the course of SARSCoV-2 infection. Compared with febrile cases, afebrile patients were more prone to develop asymptomatic and normal chest CT images. What's more, afebrile patients had a significantly higher white blood cell count, total lymphocytes, platelet count, $T$ lymphocytes, CD8+ T lymphocytes and CD4+ T lymphocytes. 
The SARS-CoV-2 virial positivity duration of the afebrile group was significantly longer than that in the febrile group. Fever and a higher count of platelet were the independent protective factors for a shorter SARS-CoV-2 RNA positivity duration.

\section{Declarations}

\section{Acknowledgement}

We thank the patients and all the medical staff in Beijing Ditan Hospital, Capital University, and thank Xinhua Ren for the critical review of a draft of this manuscript.

\section{Authors' contributions}

Study design: Rui Ding and Wen Xie. Data collection: Rugang Zhao, Ying Fan, Ligai Liu, Ying Cao, Cheng Cheng, Jing Jing Wang, Qi Wang, Yanbin Wang, Ting Zhang and Minghui LI. Data analysis: Rui Ding and Rugang Zhao. Writing: Rui Ding, Rugang Zhao and Wen Xie. All authors read and approved the final manuscript. Rui Ding and Rugang Zhao contributed equally to this work. All authors read and approved the final manuscript.

\section{Funding}

This research did not receive any specific grant from funding agencies in the public, commercial, or notfor-profit sectors.

\section{Availability of data and materials}

The datasets used and/or analyzed during the current study are available from the corresponding author on reasonable request.

\section{Ethical approval and consent to participate}

This retrospective study was approved by the ethics committee of Beijing Ditan Hospital, Capital Medical University (No. 202000201). Written or oral informed consent was obtained from patients. 


\section{Conflict of interest}

We declare no conflict of interest.

\section{Authors details}

${ }^{1}$ Center of Liver Disease, Beijing Ditan Hospital, Capital Medical University, Beijing, 100015, China

${ }^{2}$ Beijing Key Laboratory of Emerging Infectious Diseases, Beijing Ditan Hospital, Capital Medical University, Beijing, 100015, China

${ }^{3}$ Department of Orthopedic, Beijing Ditan Hospital, Capital Medical University, Beijing, 100015, China

\section{Abbreviations}

ICU: Intensive Care Unit; RNA: ribonucleic acid; CT: computed tomography

\section{References}

1. Who. WHO Director-General's remarks at the media briefing on 2019-nCoV on 11 February 2020. 2020. https://www.who.int/dg/speeches/detail/who-director-general-s-remarks-at-the-media-briefingon-2019-ncov-on-11-february-2020.

2. Who. Coronavirus disease 2019 (COVID-19) Situation Report - 97. 2020. https://www.who.int/docs/default-source/coronaviruse/situation-reports/20200426-sitrep-97-covid19.pdf?sfvrsn=d1c3e800_6.

3. Huang C, Wang Y, Li X, Ren L, Zhao J, Hu Y, et al. Clinical features of patients infected with 2019 novel coronavirus in Wuhan, China. Lancet. 2020;395(10223):497-506.

4. Wang D, Hu B, Hu C, Zhu F, Liu X, Zhang J, et al. Clinical Characteristics of 138 Hospitalized Patients With 2019 Novel Coronavirus-Infected Pneumonia in Wuhan, China. Jama. 2020;323(11):1061-9.

5. Zhu N, Zhang D, Wang W, Li X, Yang B, Song J, et al. A Novel Coronavirus from Patients with Pneumonia in China, 2019. N Engl J Med. 2020;382(8):727-33.

6. China NHCOTPSRO. Guidelines for The Diagnosis and Treatment of Novel Coronavirus (2019-nCoV) Infection by The National Health Commission (trial version 7). 2020. 
7. Jin YH, Cai L, Cheng ZS, Cheng H, Deng T, Fan YP, et al. A rapid advice guideline for the diagnosis and treatment of 2019 novel coronavirus (2019-nCoV) infected pneumonia (standard version). Mil Med Res. 2020;7(1):4.

8. Who. WHO Director-General's opening remarks at the Mission briefing on COVID-19-12 March 2020. 2020. https://www.who.int/dg/speeches/detail/who-director-general-s-opening-remarks-at-themission-briefing-on-covid-19--12-march-2020.

9. Hui DS, Wong PC, Wang C. SARS: clinical features and diagnosis. Respirology. 2003;8 Suppl(Suppl 1):S20-4.

10. Zumla A, Hui DS, Perlman S. Middle East respiratory syndrome. Lancet. 2015;386(9997):995-1007.

11. Guan WJ, Ni ZY, Hu Y, Liang WH, Ou CQ, He JX, et al. Clinical Characteristics of Coronavirus Disease 2019 in China. N Engl J Med. 2020;382(18):1708-20.

12. Villalonga N, David M, Bielanska J, Vicente R, Comes N, Valenzuela C, et al. Immunomodulation of voltage-dependent $\mathrm{K}+$ channels in macrophages: molecular and biophysical consequences. J Gen Physiol. 2010;135(2):135-47.

13. Janeway CA, Jr. How the immune system protects the host from infection. Microbes Infect. 2001;3(13):1167-71.

14. Walter EJ, Hanna-Jumma S, Carraretto M, Forni L. The pathophysiological basis and consequences of fever. Crit Care. 2016;20(1):200.

15. Evans SS, Repasky EA, Fisher DT. Fever and the thermal regulation of immunity: the immune system feels the heat. Nat Rev Immunol. 2015;15(6):335-49.

16. Chen Q, Wang WC, Bruce R, Li H, Schleider DM, Mulbury MJ, et al. Central role of IL-6 receptor signaltransducing chain gp130 in activation of L-selectin adhesion by fever-range thermal stress. Immunity. 2004;20(1):59-70.

17. Franco AT, Corken A, Ware J. Platelets at the interface of thrombosis, inflammation, and cancer. Blood. 2015;126(5):582-8.

18. Koupenova M, Clancy L, Corkrey HA, Freedman JE. Circulating Platelets as Mediators of Immunity, Inflammation, and Thrombosis. Circ Res. 2018;122(2):337-51.

19. Lee N, Hui D, Wu A, Chan P, Cameron P, Joynt GM, et al. A major outbreak of severe acute respiratory syndrome in Hong Kong. N Engl J Med. 2003;348(20):1986-94.

20. Jenne CN, Kubes P. Platelets in inflammation and infection. Platelets. 2015;26(4):286-92.

21. Weyrich AS, Zimmerman GA. Platelets: signaling cells in the immune continuum. Trends Immunol. 2004;25(9):489-95.

22. Assinger A. Platelets and infection - an emerging role of platelets in viral infection. Front Immunol. 2014;5:649.

23. Chabert A, Hamzeh-Cognasse H, Pozzetto B, Cognasse F, Schattner M, Gomez RM, et al. Human platelets and their capacity of binding viruses: meaning and challenges? BMC Immunol. 2015;16:26. 
24. Morrell CN, Aggrey AA, Chapman LM, Modjeski KL. Emerging roles for platelets as immune and inflammatory cells. Blood. 2014;123(18):2759-67.

25. Jenne CN, Urrutia R, Kubes P. Platelets: bridging hemostasis, inflammation, and immunity. Int J Lab Hematol. 2013;35(3):254-61.

26. Ali RA, Wuescher LM, Worth RG. Platelets: essential components of the immune system. Curr Trends Immunol. 2015;16:65-78.

27. Chaipan C, Soilleux EJ, Simpson P, Hofmann H, Gramberg T, Marzi A, et al. DC-SIGN and CLEC-2 mediate human immunodeficiency virus type 1 capture by platelets. J Virol. 2006;80(18):8951-60.

28. Kullaya VI, De Mast Q, Van Der Ven A, Elmoussaoui H, Kibiki G, Simonetti E, et al. Platelets Modulate Innate Immune Response Against Human Respiratory Syncytial Virus In Vitro. Viral Immunol. 2017;30(8):576-81.

29. Mcmorran BJ, Marshall VM, De Graaf C, Drysdale KE, Shabbar M, Smyth GK, et al. Platelets kill intraerythrocytic malarial parasites and mediate survival to infection. Science. 2009;323(5915):797800 .

30. Zhao H, Li S, Yang R. Thrombocytopenia in patients with systemic lupus erythematosus: significant in the clinical implication and prognosis. Platelets. 2010;21(5):380-5.

31. Wong SF, Chow KM, De Swiet M. Severe Acute Respiratory Syndrome and pregnancy. Bjog. 2003;110(7):641-2.

32. Assiri A, Al-Tawfiq JA, Al-Rabeeah AA, Al-Rabiah FA, Al-Hajjar S, Al-Barrak A, et al. Epidemiological, demographic, and clinical characteristics of 47 cases of Middle East respiratory syndrome coronavirus disease from Saudi Arabia: a descriptive study. Lancet Infect Dis. 2013;13(9):752-61.

33. Su R, Li Z, Wang Y, Liu Y, Zheng X, Gao C, et al. Imbalance between Th17 and regulatory T cells in patients with systemic lupus erythematosus combined EBV/CMV viraemia. Clin Exp Rheumatol. 2019.

34. Cui W, Fan Y, Wu W, Zhang F, Wang JY, Ni AP. Expression of lymphocytes and lymphocyte subsets in patients with severe acute respiratory syndrome. Clin Infect Dis. 2003;37(6):857-9.

35. Li T, Qiu Z, Zhang L, Han Y, He W, Liu Z, et al. Significant changes of peripheral T lymphocyte subsets in patients with severe acute respiratory syndrome. J Infect Dis. 2004;189(4):648-51.

36. Wang F, Nie J, Wang H, Zhao Q, Xiong Y, Deng L, et al. Characteristics of Peripheral Lymphocyte Subset Alteration in COVID-19 Pneumonia. J Infect Dis. 2020;221(11):1762-9.

37. Yoshikawa T, Hill T, Li K, Peters CJ, Tseng CT. Severe acute respiratory syndrome (SARS) coronavirusinduced lung epithelial cytokines exacerbate SARS pathogenesis by modulating intrinsic functions of monocyte-derived macrophages and dendritic cells. J Virol. 2009;83(7):3039-48.

38. Yu F, Yan L, Wang N, Yang S, Wang L, Tang Y, et al. Quantitative Detection and Viral Load Analysis of SARS-CoV-2 in Infected Patients. Clin Infect Dis. 2020;71(15):793-8.

\section{Figures}




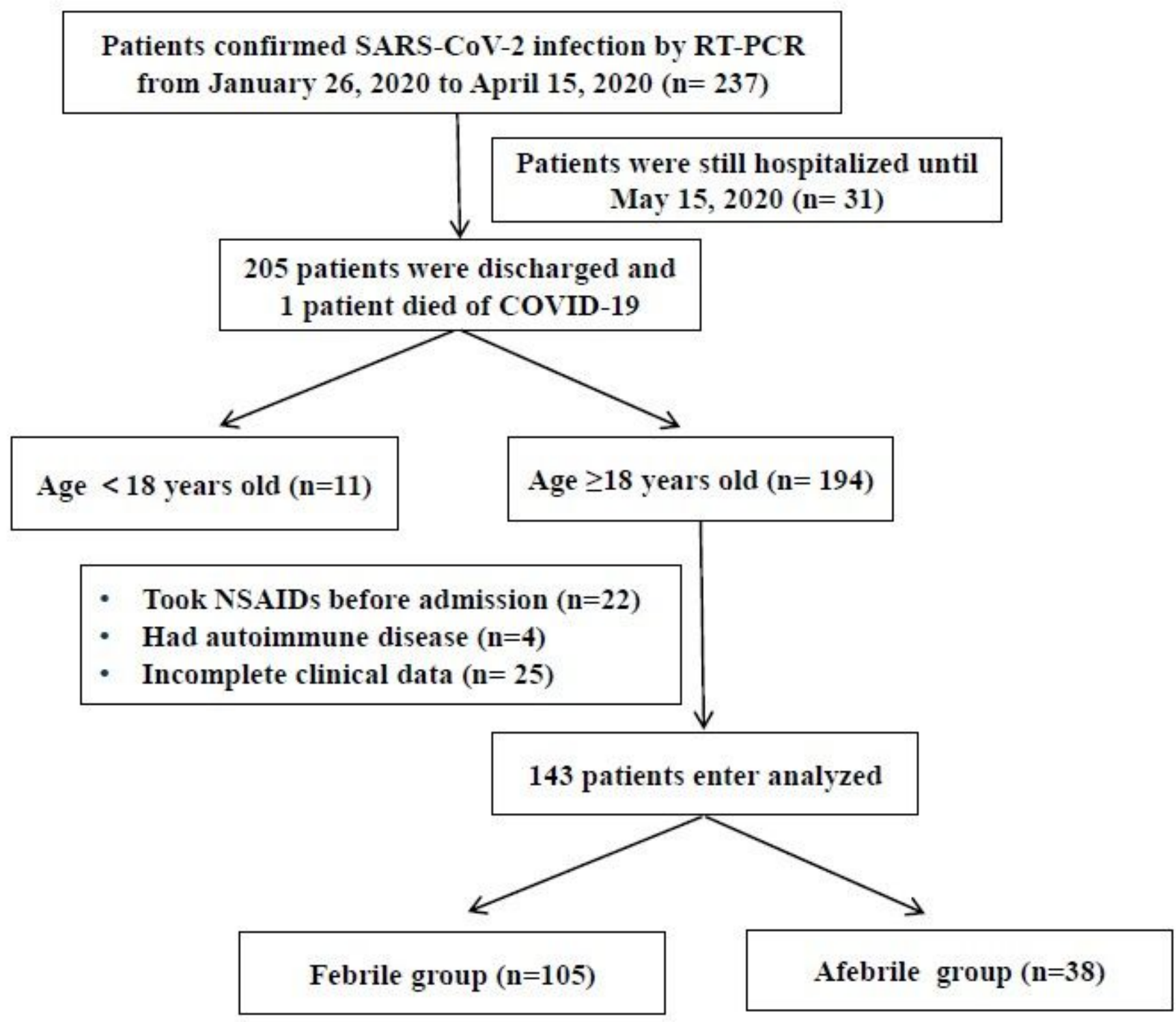

Figure 1

The flow of patient enrollment. Legends: From January 26, 2020 to April 15, 2020, 237 patients were admitted to Beijing Ditan Hospital, Capital Medical University. 143 patients were enrolled into this study at last. 


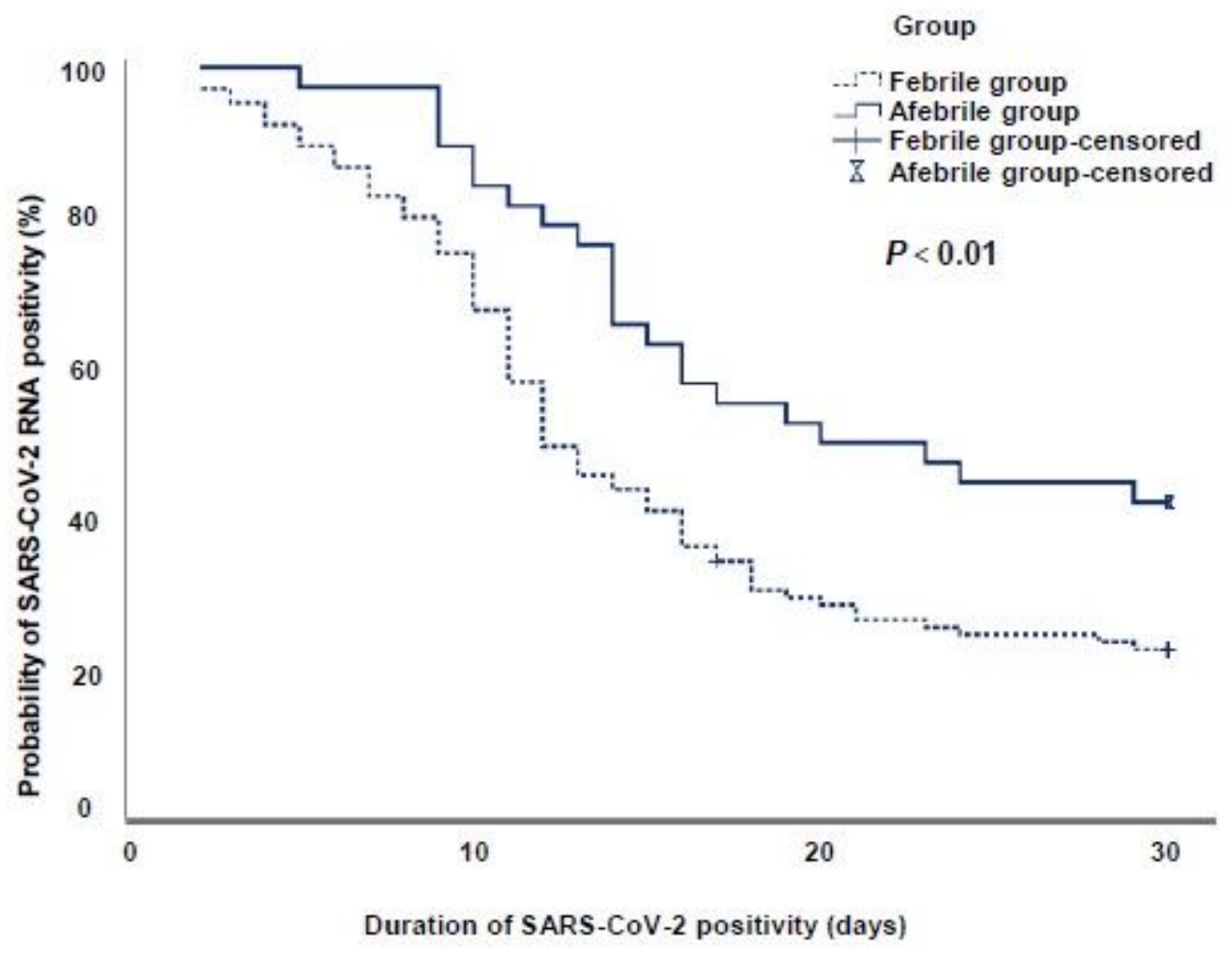

Figure 2

The survival between groups with and without fever. Legends: Kaplan-Meier method with Log-rank test was performed to evaluate the significance of fever for the duration of SARS-CoV-2 viral positivity ( $\mathrm{P} \otimes$ $0.01)$. 